Original Article

\title{
Correlations among pelvic positions and differences in lower extremity joint angles during walking in female university students
}

\author{
Misuk Cho, $\mathrm{PhD}, \mathrm{PT}^{1)}$ \\ 1) Department of Physical Therapy, Korea Nazarene University: Wolbong Ro 48, Seobuk-gu, Cheonan- \\ si, Chungcheongnam-do 330-718, Republic of Korea
}

\begin{abstract}
Purpose] This study aimed to identify correlations among pelvic positions and differences in lower extremity joint angles during walking in female university students. [Subjects] Thirty female university students were enrolled and their pelvic positions and differences in lower extremity joint angles were measured. [Methods] Pelvic position, pelvic torsion, and pelvic rotation were assessed using the BackMapper. In addition, motion analysis was performed to derive differences between left and right flexion, abduction, and external rotation ranges of hip joints; flexion, abduction, and external rotation ranges of knee joints; and dorsiflexion, inversion, and abduction ranges of ankle joints, according to X, Y, and Z-axes. [Results] Pelvic position was found to be positively correlated with differences between left and right hip flexion $(\mathrm{r}=0.51)$, hip abduction $(\mathrm{r}=0.62)$, knee flexion $(\mathrm{r}=0.45)$, knee abduction $(\mathrm{r}=0.42)$, and ankle inversion $(\mathrm{r}=0.38)$. In addition, the difference between left and right hip abduction showed a positive correlation with difference between left and right ankle dorsiflexion $(r=0.64)$. Moreover, differences between left and right knee flexion exhibited positive correlations with differences between left and right knee abduction $(\mathrm{r}=0.41)$ and ankle inversion $(\mathrm{r}=0.45)$. [Conclusion] Bilateral pelvic tilt angles are important as they lead to bilateral differences in lower extremity joint angles during walking.

Key words: Pelvic position, Lower extremity joint angle, Walking
\end{abstract}

(This article was submitted Feb. 13, 2015, and was accepted Mar. 7, 2015)

\section{INTRODUCTION}

Walking, which can be defined as moving the body to a target spot or destination using two legs, is a complex and integrated activity requiring low energy consumption ${ }^{1)}$. Although it appears to be an easy-to-perform task, it actually requires comprehensive use of all nerves and skeletal muscles. Furthermore, learning to walk requires considerable practice and effort and takes at least four years to master ${ }^{2}$. The gait cycle is divided into stance and swing phases. During the stance phase, the foot is in contact with the ground, whereas during the swing phase, the same foot lifts off the ground and moves forward. These two main phases can be further divided into lower-level phases. The stance phase is divided into heel strike, foot flat, mid-stance, and toe off, whereas the swing phase is divided into acceleration, mid-swing, and deceleration $^{3)}$.

The pelvis is a structure located between the hip joint and lumbosacral region. With the use of multiple muscles, the pelvis controls the movements of the hip joint and

Corresponding author. Misuk Cho (E-mail: mscho@kornu. ac.kr)

C2015 The Society of Physical Therapy Science. Published by IPEC Inc. This is an open-access article distributed under the terms of the Creative Commons Attribution Non-Commercial No Derivatives (by-ncnd) License $<$ http://creativecommons.org/licenses/by-nc-nd/3.0/>. lumbosacral region. In this respect, the pelvic position is the most important factor for determining the body's sagittal alignment and posture ${ }^{4)}$. With the pelvis in the neutral position, correct posture can be maintained, and movements occurring during activities of daily living and gait can be improved via proper control of the upper and lower extremities in dynamic postures ${ }^{5)}$. In addition, mechanical problems in the musculoskeletal system of the lumbar vertebrae can occur depending on the location of the pelvis. Moreover, forward and backward pelvic tilt exercises can influence spinal stability and physical alignment. Therefore, pelvic position is an important consideration duringwalking ${ }^{6}$.

Several studies have been conducted on pelvic stability and postures required during walking. Lumbo-pelvic stabilization and posture have been shown to improve with bridge exercises ${ }^{7)}$ and various mat exercises ${ }^{8)}$. In addition, some studies have examined postures ${ }^{9}$ or gait variables ${ }^{10)}$ through pelvic adjustment. However, none of the studies have addressed the correlations among pelvic positions and differences in lower-extremity joint angles during walking. This basic data, which will be helpful for future studies on walking

\section{SUBJECTS AND METHODS}

Thirty female students at the $\mathrm{K}$ University were selected as subjects. The average (mean \pm standard deviation) age, height, and weight of the subjects were $22.3 \pm 3.4$ years, 
Table 1. Mean \pm Standard deviation of PPO, PTO, PRO, HXD, HYD, HZD, KXD, KYD, KZD, AXD, AYD, and AZD (Unit-degree)

\begin{tabular}{|c|c|c|c|c|c|c|c|c|c|c|c|}
\hline PPO & PTO & PRO & HXD & HYD & HZD & KXD & KYD & KZD & AXD & AYD & AZD \\
\hline $2.7 \pm 0.9$ & $3.6 \pm 1.7$ & $2.2 \pm 1.5$ & $5.2 \pm 1.8$ & $3.5 \pm 1.7$ & $5.1 \pm 1.4$ & $6.3 \pm 1.6$ & $6.4 \pm 1.6$ & $6.2 \pm 1.4$ & $5.8 \pm 1.5$ & $5.5 \pm 1.0$ & $5.8 \pm 1.4$ \\
\hline
\end{tabular}

$163.2 \pm 5.4 \mathrm{~cm}$, and $53.5 \pm 5.7 \mathrm{~kg}$, respectively. The exclusion criteria were as follows: musculoskeletal or neurological problems; lumbar and pelvic pain during daily activities; incorrect posture due to scars related to previous burns or surgeries; and inability to walk normally. This study was approved by the University's Institutional Review Board, and the subjects were safely protected during all experimental procedures. All the subjects understood the purpose of this study and provided written informed consent prior to their participation, in accordance with the ethical standards of the Declaration of Helsinki.

This study measured the pelvic positions and differences in lower-extremity joint angles during walking. The BackMapper (ABW, Germany), a three-dimensional spinal diagnostic system, was used to measure pelvic positions. The BackMapper is an apparatus that obtains precise measurements of the spinal form, location, and degree of distortion, evaluating the spinal form from the backward, forward, downward, and upward directions. It analyzes the inclination of the coronal plane, pelvic position (PPO), left and right inclination of the pelvis, pelvic torsion (PTO), degree of declination of the hip bone, and pelvic rotation (PRO).

The Qualisys Track Manager (Qualisys AB, Sweden), a three-dimensional wireless motion analysis system with six cameras operating at $100 \mathrm{~Hz}$ per second, was used to analyze the kinematic motions of lower extremities in the subjects during walking. The L-frame was fixed on the starting point of walking to set the spatial coordinates. In terms of directions, the upper vertical axis was set as the $+Z$-axis, and the direction of motions and the horizontal axis were set as the + Y-axis and $+\mathrm{X}$-axis, respectively. Based on the L-frame, the space within which movements would be performed was defined using the T-Wand to recognize markers clearly. Reflective markers for static postures to form basic frames were attached to each side of the sacrum, anterior superior iliac spine, thigh, and shank. Four markers were attached to each side of the thigh and shank in a square form to create a three-dimensional space. In addition, reflective markers were attached to the medial and lateral epicondyles, lateral malleolus, and medial malleolus on both sides of the body. In the segments of the foot, reflective markers were attached to specific areas of the foot surface, which corresponded with the calcaneal tuberosity and the first and fifth metatarsal heads. To track the three-dimensional motions of three lower extremity joints during dynamic postures, the markers for static postures, which had been attached to the medial and lateral epicondyles, lateral malleolus, and medial malleolus, were removed prior to filming of the respective motions. To reduce errors, a single well-trained person attached these reflective markers. Motion analysis was performed to derive differences between left and right flexion, abduction, and external rotation range of motion (ROM) of hip joints, flexion, abduction, and external rotation ROM of knee joints, and dorsiflexion, inversion, and abduction ROM of ankle joints, according to the $\mathrm{X}, \mathrm{Y}$, and $\mathrm{Z}$-axes.

The measured data were analyzed using the SPSS 12.0 KO (SPSS, Chicago, IL, USA) software, and the collected data are presented in terms of means and standard deviations. Pearson's correlation coefficient was used to observe correlations among the PPO, PTO, PRO, differences between left and right hip flexion (HXD), differences between left and right hip abduction (HYD), differences between left and right hip external rotation (HZD), differences between left and right knee flexion (KXD), differences between left and right knee abduction (KYD), differences between left and right knee external rotation (KZD), differences between left and right ankle dorsiflexion (AXD), differences between left and right ankle inversion (AYD), and differences between left and right ankle abduction (AZD). The statistical significance level was set at $\alpha=0.05$.

\section{RESULTS}

The PPO, PTO, PRO, HXD, HYD, HZD, KXD, KYD, $\mathrm{KZD}, \mathrm{AXD}$, AYD, and AZD measurements are presented as mean \pm standard deviation in Table 1 . In terms of correlations among these variables, PPO showed positive correlations with HXD, HYD, KXD, KYD, and AYD. In other words, an increase in PPO resulted in corresponding increases in HXD $(\mathrm{r}=0.51)$, HYD $(\mathrm{r}=0.62), \mathrm{KXD}(\mathrm{r}=0.45), \mathrm{KYD}(\mathrm{r}=0.42)$, and AYD ( $\mathrm{r}=0.38)$. HXD exhibited a positive correlation with $\mathrm{AXD}$, as an increase in HXD resulted in a corresponding increase in AXD ( $\mathrm{r}=0.64)$. KXD also showed positive correlations with KYD and $\mathrm{AYD}$; an increase in KXD led to corresponding increases in KYD $(r=0.41)$ and AYD $(r=0.45)$, as shown in Table 2.

\section{DISCUSSION}

Humans are exposed to gravity during upright walking. This causes them to experience pelvic and lower-extremity misalignment, which in turn influence posture, gait patterns, and balance. The pelvis supports the abdomen and connects the vertebrae and lower extremities. When an individual stands up from a seated position, the pelvis transfers the body weight from the vertebrae to the lower extremities and 
Table 2. Correlations among PPO, PTO, PRO, HXD, HYD, HZD, KXD, KYD, KZD, AXD, AYD, and AZD

\begin{tabular}{|c|c|c|c|c|c|c|c|c|c|c|c|c|}
\hline Category & PPO & PTO & PRO & HXD & HYD & HZD & KXD & KYD & KZD & AXD & AYD & AZD \\
\hline PPO & 1 & & & & & & & & & & & \\
\hline PTO & 0.18 & 1 & & & & & & & & & & \\
\hline PRO & -0.01 & 0.12 & 1 & & & & & & & & & \\
\hline HXD & $0.51^{* *}$ & -0.02 & -0.30 & 1 & & & & & & & & \\
\hline HYD & $0.62 *$ & 0.02 & -0.02 & 0.26 & 1 & & & & & & & \\
\hline HZD & 0.37 & 0.11 & -0.17 & 0.15 & 0.08 & 1 & & & & & & \\
\hline KXD & $0.45^{*}$ & 0.25 & 0.03 & 0.21 & 0.00 & 0.34 & 1 & & & & & \\
\hline KYD & $0.42 *$ & 0.04 & 0.02 & 0.32 & 0.31 & 0.00 & $0.41^{*}$ & 1 & & & & \\
\hline KZD & 0.15 & -0.28 & 0.11 & 0.10 & 0.04 & -0.19 & -0.10 & 0.15 & 1 & & & \\
\hline AXD & 0.19 & 0.00 & -0.12 & $0.64 * *$ & -0.03 & 0.06 & 0.14 & 0.19 & 0.10 & 1 & & \\
\hline AYD & $0.38^{*}$ & 0.19 & -0.15 & 0.11 & 0.25 & 0.22 & $0.45^{*}$ & 0.09 & 0.25 & -0.15 & 1 & \\
\hline AZD & 0.10 & $-0,00$ & 0.11 & -0.24 & -0.07 & 0.25 & 0.00 & -0.11 & 0.16 & -0.29 & 0.13 & 1 \\
\hline
\end{tabular}

** $\mathrm{p}<0.01, * \mathrm{p}<0.05$

PPO: pelvic position, PTO: pelvic torsion, PRO: pelvic rotation, HXD: difference between left and right hip flexion, HYD: difference between left and right hip abduction, HZD: difference between left and right hip external rotation, KXD: difference between left and right knee flexion, KYD: difference between left and right knee abduction, KZD: difference between left and right knee external rotation, AXD: difference between left and right ankle dorsiflexion, AYD: difference between left and right ankle inversion, AZD: difference between left and right ankle abduction

maintains correct posture, thereby enabling smooth movement of the upper extremities ${ }^{11)}$. With the pelvis positioned in neutral, correct posture can be maintained as well as appropriate motions can be performed during daily living activities; additionally, gait ability can be improved via proper control of the upper and lower extremities during dynamic postures $^{12)}$.

In a similar study on the pelvis and walking, Cho and Jun selected 30 female university students and randomly divided them into a pelvic adjustment group $(n=15)$ and a stretching group $(\mathrm{n}=15)$, which served as the control group, and analyzed their gait. They reported that differences in step length, stance phase, swing phase, single support, and step time were reduced with pelvic adjustment ${ }^{10)}$. Moreover, Cho selected 30 female university students and randomly divided them into a pelvic adjustment group $(\mathrm{n}=15)$ and a stretching group $(\mathrm{n}=15)$. After analyzing their postures, the author reported that pelvic adjustment showed statistically significant effects on trunk imbalance, PPO, PTO, and the position of the scapulae ${ }^{9}$ ). In addition, a study reported that trunk stabilization exercises enhanced normal gait patterns in stroke patients and their perception of the affected lower extremity, promoting normal exercise modes and reducing excessive muscle tension by increasing the symmetry of pelvic alignment ${ }^{13)}$.

Normal position and stability of the pelvis are important for balanced walking. Several existing studies have conducted gait analyses after various interventions in patients with hemiplegia ${ }^{14)}$ or cerebral palsy ${ }^{15)}$ by employing threedimensional gait analysis techniques. However, few studies have addressed correlations among pelvic positions and differences in lower-extremity joint angles during walking. The present study analyzed correlations among PPO, PTO, PRO, HXD, HYD, HZD, KXD, KYD, KZD, AXD, AYD, and AZD. The results of this correlation analysis are as follows. PPO showed positive correlations with HXD, HYD,
$\mathrm{KXD}, \mathrm{KYD}$, and AYD; in other words, an increase in PPO resulted in corresponding increases in HXD, HYD, KXD, KYD, and AYD. In addition, HXD showed a positive correlation with $\mathrm{AXD}$, in which an increase in HXD led to a corresponding increase in AXD. Moreover, KXD exhibited positive correlations with KYD and AYD, as an increase in KXD resulted in corresponding increases in KYD and AYD.

To summarize these findings, increases in left and right pelvic tilt resulted in corresponding increases in differences between left and right hip flexion, hip abduction, knee flexion, knee abduction, and ankle inversion. This may have resulted from changes in weight support and lower-extremity tilt according to changes in pelvic height on the left and right sides. Therefore, both left and right pelvic tilt were important factors that led to differences between the left and right lower-extremity joint angles during walking. When these types of differences are present, pelvic adjustment is considered necessary for balanced walking. Additional studies should be carried out to implement pelvic adjustment or lumbo-pelvic stabilization exercises as interventions to reduce differences between the left and right lower extremity joint angles during walking.

\section{ACKNOWLEDGEMENT}

This research was supported by the Korea Nazarene University Research Grants in 2015.

\section{REFERENCES}

1) Perry J, Garrett M, Gronley JK, et al.: Classification of walking handicap in the stroke population. Stroke, 1995, 26: 982-989. [Medline] [CrossRef]

2) Eckert H: Motor development. Indianapolis: Benchaak Press, 1987.

3) Vaughan CL, Davis BL, O'Connor JC: Dynamics of human gait, 2nd ed. South Africa: Kiboho Publishers, 1992.

4) Vaz G, Roussouly P, Berthonnaud E, et al.: Sagittal morphology and equilibrium of pelvis and spine. Eur Spine J, 2002, 11: 80-87. [Medline] 
[CrossRef]

5) Bae SS, Kim TY, Jung HA, et al.: Discussion of the pelvis kinematics Korean Soc Phys Ther, 1999, 11: 93-102.

6) Walsh M, Connolly P, Jenkinson A, et al.: Leg length discrepancy-an experimental study of compensatory changes in three dimensions using gait analysis. Gait Posture, 2000, 12: 156-161. [Medline] [CrossRef]

7) García-Vaquero MP, Moreside JM, Brontons-Gil E, et al.: Trunk muscle activation during stabilization exercises with single and double leg support. J Electromyogr Kinesiol, 2012, 22: 398-406. [Medline] [CrossRef]

8) Liebenson C: Spinal stabilization training: the transverse abdominus. Journal of Bodywork and Movement Therapies, 1998, 2: 218-223. [CrossRef]

9) Cho M: The influence of pelvic adjustment on the posture of female university students. J Phys Ther Sci, 2013, 25: 785-787. [Medline] [CrossRef]

10) Cho M, Jun I: Effects of pelvic adjustment on female university students' gait variables. J Phys Ther Sci, 2014, 26: 759-762. [Medline] [CrossRef] 11) Magee DJ: Orthopedic Physical Assessment, 5th ed. St. Louis: Elsevier Inc, 2008.

12) Kapandji IA: Physiology of the joints, 6th ed. Philadelphia: Churchill Livingstone, 2007.

13) Trueblood M: Rehabilitation of Gait in chronic stroke patients. Phys Ther, 2003, 83: 566-581.

14) Defrin R, Ben Benyamin S, Aldubi RD, et al.: Conservative correction of leg-length discrepancies of $10 \mathrm{~mm}$ or less for the relief of chronic low back pain. Arch Phys Med Rehabil, 2005, 86: 2075-2080. [Medline] [CrossRef]

15) Bonnyaud C, Pradon D, Zory R, et al.: Effects of a gait training session combined with a mass on the non-paretic lower limb on locomotion of hemiparetic patients: a randomized controlled clinical trial. Gait Posture, 2013, 37: 627-630. [Medline] [CrossRef] 